\title{
Unusual occurrence of hemophagocytic lymphohistiocytosis in HIV-positive person with visceral leishmaniasis
}

\author{
Ikbel Kooli, Wafa Marrakchi, Adnene Toumi, Abir Aouam, Hammouda Baba, Mohamed Chakroun
}

Fattouma Bourguiba Hospital, Tunisia

\begin{abstract}
Introduction: Visceral leishmaniasis is a well-recognized opportunistic infection in people living with HIV (PLHIV). Unlike adults, in children this infection is frequently associated with hemophagocytic lymphohistiocytosis (HLH). We report a case of HLH in HIV-positive person with visceral leishmaniasis. Case description: A 25-year-old man known living with HIV since 2013 was admitted to infectious diseases department in March 2017. His clinical examination was clear. His initial viral load was 630,000 copies $/ \mathrm{mm}^{3}$ and CD4+ cells count was $12 / \mathrm{mm}^{3}$. No opportunistic infections were noted. The patient was started on antiretroviral therapy. During hospitalization, he developed fever, asthenia, rhinorrhea, and odynophagia. The diagnosis of HLH was retained because of pancytopenia, cytolysis, hyponatremia, high level of ferrinemia, and hemophagocytosis. Etiological investigations revealed positive Leishmania PCR. Also, Leishmania was detected in sternal puncture. Patient received meglumine antimoniate (glucantime) $20 \mathrm{mg} / \mathrm{kg} /$ day for 21 days with favorable outcomes. To prevent relapse, he received meglumine antimoniate $20 \mathrm{mg} / \mathrm{kg} / \mathrm{month}$ as long as his CD4+ count was less than 100 cells $/ \mathrm{mm}^{3}$. After 1-year follow up, no relapse was detected.

Conclusions: Clinical and laboratory presentation of visceral leishmaniasis in PLHIV may differ from classic kala-azar. In our case, HLH was the reason for VL discovery.
\end{abstract}

HIV AIDS Rev 2021; 20, 4: 311-313 DOI: https://doi.org/10.5114/hivar.2021.111899

Key words: HIV, hemophagocytic lymphohistiocytosis, visceral leishmaniasis.

\section{Introduction}

Visceral leishmaniasis (VL) is a vector-borne parasitic disease, caused by Leishmania donovani species complex, predominantly affecting macrophages [1]. It is a well-recognized opportunistic infection in people living with HIV (PLHIV). Majority of HIV-Leishmania co-infected cases show classic features of VL. Hemophagocytic lymphohistiocytosis (HLH) is a rare reactive hyperplastic disease of mononuclear phagocytic cell system. It is a life-threatening syndrome, clinically divided into 2 types: primary HLH and secondary HLH. Infection is the most common etiology underlying

Address for correspondence: Dr. Ikbel Kooli, Fattouma Bourguiba Hospital, Tunisia, e-mail: kooli.ikbel@gmail.com

secondary HLH [2], which can be induced by viruses, bacteria, fungi, parasites, and protozoa. It is frequently associated with VL in immunocompetent children. For PLHIV, this syndrome is rarely associated with VL. In the present paper, we describe a patient with stage $3 \mathrm{HIV}$ infection and atypical features of VL.

\section{Case description}

A 25-year-old man was known living with HIV since 2013 and was lost to follow-up. He was admitted in our de-

Article history:

Received: 18.06.2020

Received in revised form: 21.03 .2021

Accepted: 23.03.2021

Available online: 30.11 .2021
International Journal of HIV-Related Problems

HIV \& AIDS

R e v i e w 

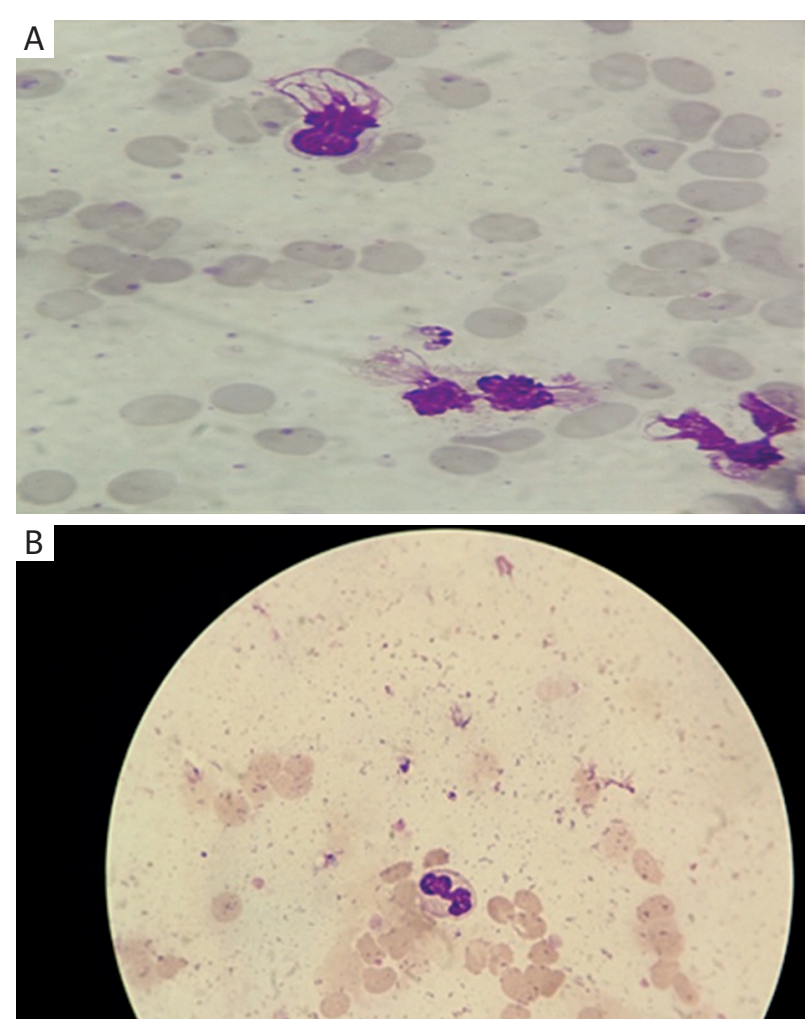

Figure 1. Leishmania in sternal puncture

partment in March 2017 to manage his infection. His clinical examination was clear. The patent's initial viral load was 630,000 copies $/ \mathrm{mm}^{3}$ and CD4+ cells count was $12 / \mathrm{mm}^{3}$. No opportunistic infections were noted. The patient was started on antiretroviral therapy. During hospitalization, he developed fever, asthenia, rhinorrhea, and odynophagia. Severe pancytopenia was shown in blood samples, including total white blood cell count of $900 / \mathrm{mm}^{3}$, neutrophils of $600 / \mathrm{mm}^{3}$, hemoglobin of $7.2 \mathrm{~g} / \mathrm{dl}$, and platelet count of $62,000 \mathrm{~mm}^{3} / 1$.

His liver function test showed serum ALT $=97 \mathrm{U} / \mathrm{l}$ and $\mathrm{AST}=40 \mathrm{U} / \mathrm{l}$. He had hyponatremia at $130 \mathrm{mmol} / \mathrm{l}$, cholesterol level of $2.4 \mathrm{mmol} / \mathrm{l}$, and a high level of ferrinemia of 2,000 ng/ml. A sternal puncture had revealed hemophagocytosis. Diagnosis of hemophagocytic lymphohistiocytosis $(\mathrm{HLH})$ was retained. Etiological investigation revealed positive Leishmania PCR. Also, Leishmania was detected in sternal puncture. Because of pancytopenia, amphotericin B could not be initiated, and the patient received meglumine antimoniate (glucantime) $20 \mathrm{mg} / \mathrm{kg} /$ day for 21 days with favorable outcomes. To prevent a relapse, he receive meglumine antimoniate $20 \mathrm{mg} / \mathrm{kg} / \mathrm{month}$ as long as his CD4+ count was less than 100 cells $/ \mathrm{mm}^{3}$. After 1-year follow-up, no relapse was observed.

\section{Discussion}

In the current paper, we described an uncommon circumstance of VL, i.e., HLH. VL can be frequently associated
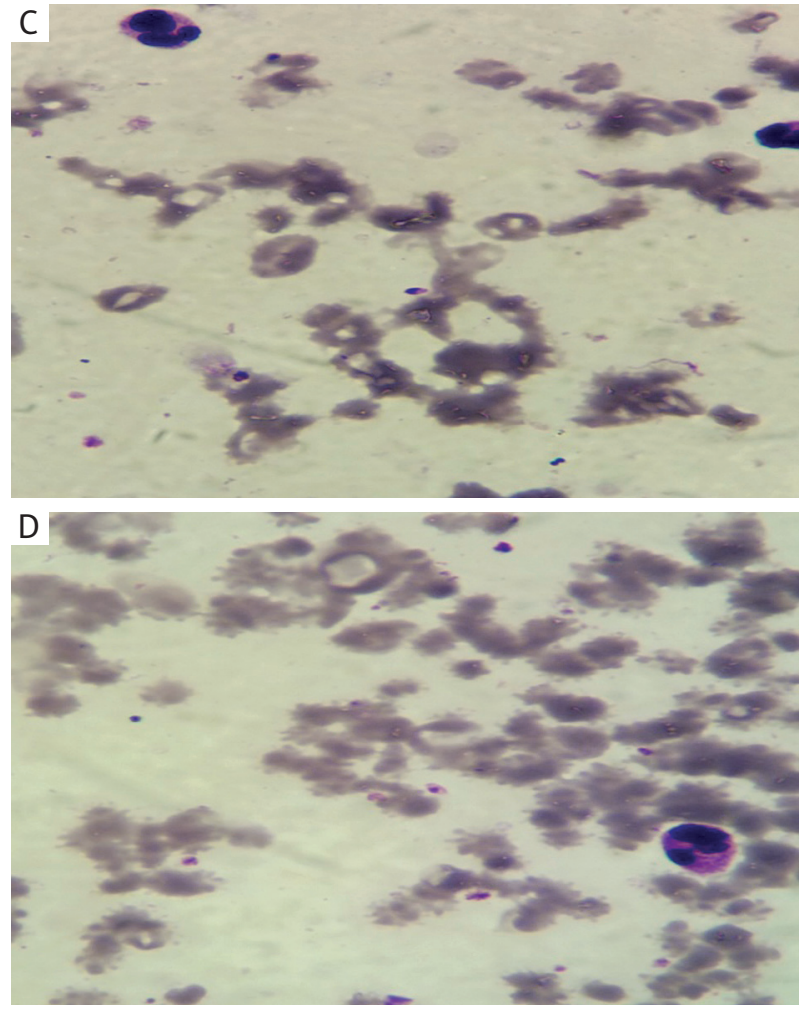

with HIV. The prevalence of patients with both HIV and VL infection in Africa is emerging [3], whereas the prevalence of HIV-VL co-infection depends on parts of the world, with $17 \%$ found in Ethiopia. Age, residence, and employment were factors associated with this co-infection [4]. In Tunisia, this HIV-VL co-infection occurrence is rare. Various clinical features are observed, including irregular bouts of fever, substantial weight loss, swelling of the spleen and liver, and anemia [5]. For PLHIV, the most frequent symptoms are weight loss, fever, and cough [6]. For our patient, it was HLH.

HLH is a systemic disease characterized by overwhelming activation of normal $\mathrm{T}$ lymphocytes and macrophages, leading to clinical and hematologic alterations. This disorder clinically manifests as fever, hepatosplenomegaly, and less frequently, lymphadenopathy, jaundice, and rash. Pancytopenia, elevated levels of serum ferritin and triglycerides, coagulopathy with hypofibrinogenemia, and abnormal liver enzymes are commonly present. This syndrome is frequent in children, and it is a common circumstance of VL in this population [7]. In adults, VL has various symptoms, but HLH is rare [7]. In our case, VL was discovered unexpectedly after bone marrow investigation due to confirmation of HLH diagnosis. Because of side effects of meglumine antimoniate and efficiency of liposomal amphotericin B (LAB), it was the first line of VL treatment. The World Health Organization recommends $\mathrm{LAB}$ as the first line treatment at a dose of $40 \mathrm{mg} / \mathrm{kg}$ in 10 doses [8].

However, in our patient due to lack of LAB and pancytopenia, the use of meglumine antimoniate was preferable. 
Several studies have shown that meglumine antimoniate caused severe adverse events (cardiotoxicity, nephrotoxicity, hepatotoxicity, and pancreatitis), resulting in high case fatality rates. Antimonies have also been shown to stimulate HIV-1 replication in vitro [4]. For our patient, no side effect was noted.

With treatment, VL usually present favorable outcome; however, in case of HIV co-infection, the outcome is poor [3]. Mortality rate is high in HIV-VL individuals [9]. This can be easily explained by pathophysiology of the two diseases, as both HIV and VL lead to a compromised immune status of a patient. Delays in diagnosis and treatment are the main causes of poor treatment outcomes. Therefore, mortality rate is comparable with stage 3/4 HIV monoinfection.

However, in our patient treated with meglumine antimoniate, the outcome was favorable.

The risk of a relapse is high in patients with CD4+ count less than 200 cells $/ \mathrm{mm}^{3}$. That is why some experts recommend secondary prophylaxis [10].

For our patient, to prevent relapse, meglumine antimoniate $20 \mathrm{mg} / \mathrm{kg} / \mathrm{month}$ was administered as long as his CD4+ count was less than 100 cells $/ \mathrm{mm}^{3}$. After 1-year follow-up, no relapse was detected.

\section{Conclusions}

HIV-VL coinfection in Tunisia is rare. Clinical and laboratory presentation of VL in HIV-co-infected patients may differ from classic kala-azar. These differences may be partly responsible for the delay in diagnosing and treating leishmaniasis. In our case, HLH was the circumstance of VL discovery. It was an unusual presentation, therefore PCR leishmaniasis must be performed in such patients, even with minor VL symptoms.

\section{Conflict of interest}

The authors have no conflict of interest.

\section{References}

1. Panda PK, Mohta S, Sharma SK, Ray A, Arava S, Vyas S. A case of pseudorheumatism with submasseteric abscess and HLH in a patient with visceral leishmaniasis: a diagnostic dilemma. J Vector Borne Dis 2016; 53: 387-390.

2. Chen L, Weng H, Li H, et al. Potential killer in the ICU - severe tuberculosis combined with hemophagocytic syndrome. Medicine (Baltimore) 2017; 96: e9142.

3. Cruz I, Nieto J, Moreno J, Canavate C, Desjeux P, Alvar J. Leishmania/HIV co-infections in the second decade. Indian J Med Res 2006; 123: 357-388.

4. Alemayehu M, Wubshet M, Mesfin N, Gebayehu A. Prevalence of human immunodeficiency virus and associated factors among visceral leishmaniasis infected patients in northwest Ethiopia: a facility based cross-sectional study. BMC Infect Dis 2017; 17: 152

5. WHO. Visceral leishmaniasis. Available at: http://www.who.int/ leishmaniasis/visceral_leishmaniasis/en/2016.

6. Lins Guedes D, Medeiros Z, da Silva ED, et al. Visceral leishmaniasis in hospitalized HIV-infected patients in Pernambuco, Brazil. Am J Trop Med Hyg 2018; 99: 1541-1546.
7. de Carvalho FHG, Lula JF, de Freitas Teles L, Prates Caldeira A, de Carvalho SFG. Hemophagocytic lymphohistiocytosis secondary to visceral leishmaniasis in an endemic area in the north of Minas Gerais, Brazil. Rev Soc Bras Med Trop 2020; 53: e20190491.

8. World Health Organization Regional Office for Europe. Manual on case management and surveillance of the leishmaniases in the WHO European Region. Denmark: World Health Organization Regional Office for Europe; 2017.

9. Diro E, Lynen L, Ritmeijer K, Boelaert M, Hailu A, van Griensven J. Visceral leishmaniasis and HIV coinfection in east Africa. PLoS Negl Trop Dis 2014; 8: e2869.

10. Monge-Maillo B, Norman FF, Cruz I, et al. Visceral leishmaniasis and HIV coinfection in the Mediterranean region. PLoS Negl Trop Dis 2014; 8: e3021. 